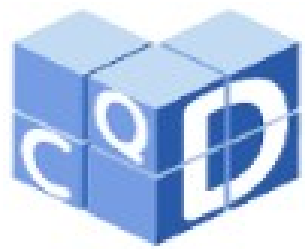

Revista Eletrônica

Paulista de Matemática

ISSN 2316-9664

Volume 17, fev. 2020

Edição Ermac

Iniciação Científica

Gabrielle Bernardo Silva

Universidade Federal de São Paulo bernardos.gabrielle@gmail.com

Raphael de Oliveira Garcia Universidade Federal de São Paulo rogarcia@unifesp.br

Jorge Andrade Costa

Universidade Federal de São Paulo jorge.costa@unifesp.br

\section{Análise de preferências do mercado automotivo e securitário através de equações diferenciais}

\author{
Analysis of automotive and insurance market preferences \\ through differential equations
}

\section{Resumo}

O propósito deste trabalho foi estudar a teoria de análise comportamental de preferências do consumidor, por meio da aplicação de equações diferenciais ordinárias em microeconomia. Para isto, supôs-se que a taxa marginal de substituição pode ser representada por uma equação diferencial ordinária, em que o lado direito de tal equação está associado a curva de indiferença. Para a modelagem matemática, considerou-se o mercado americano de automóveis e o mercado brasileiro de seguros. Com os devidos dados, foi possível obter a solução analítica do problema de valor inicial e, consequentemente, a curva de substituição entre dois produtos concorrentes do mercado considerado. As curvas de indiferença obtidas possibilitaram o entendimento sobre o comportamento de ambos os mercados, propiciando a compreensão sobre as motivações da relação de preferência dos consumidores e, assim, melhores condições para tomada de decisão.

Palavras-chave: Modelagem matemática. Equações diferenciais ordinárias. Preferências do consumidor. Comportamento de mercado. Análise financeira.

\begin{abstract}
The purpose of this paper was to apply the theory of behavioral analysis of consumer preferences, through the application of ordinary differential equations in microeconomics. For this, it was considered the marginal substitution rate can be represented by an ordinary differential equation, where the right side of equation is associated with the indifference curve. For mathematical modeling, the american vehicle market and the brazilian security market were considered. With the appropriate data, it was possible to obtain the analytical solution of the initial value problem and, consequently, the substitution curve between two competing products in the market concerned. Through the indifference curves obtained, it was possible to understand the behavior of both markets, providing an understanding of the motivations of the preference relationship of consumers and, thus, better conditions for decision making.

Keywords: Mathematical modeling. Ordinary differential equations. Consumer preferences. Market behavior. Financial analysis.
\end{abstract}




\section{Introdução}

Dentro de um mercado cada vez mais competitivo, o estudo sobre comportamento do consumidor referente as escolhas vem se intensificando a ponto de termos um ramo de estudo que engloba tal assunto, a Microeconomia. É interessante a abordagem da análise da escolha do consumidor frente a produtos de mesma utilidade, em razão da grande contribuição que pode trazer diante da concorrência presente no mercado, sendo a escolha do consumidor pelo produto fornecido pela entidade de suma importância ao resultado da mesma.

Essa escolha pode ser representada pela taxa marginal de substituição que se refere ao máximo de unidades que um consumidor está disposto a deixar de consumir, por uma unidade adicional de um outro bem. A fórmula que associa diferentes níveis de utilidade é denominada função utilidade, sendo composta pela combinação de todas as cestas de mercado que forneçam o mesmo nível de utilidade, as curvas de indiferença [1].

O entendimento do que estimula um indivíduo a escolher entre dois produtos de mesma utilidade é de grande interesse para as empresas, sendo denominada como Teoria do Consumidor. Essa teoria microeconômica busca compreender os motivos pelos quais um indivíduo realiza sua opção de consumo, sendo as influências internas e externas aspectos extremamente decisivos para o comportamento praticado.

Em continuação à linha de pesquisa do trabalho apresentado no VI ERMAC em Bauru [2], para analisar as preferências do consumidor utilizando equações diferenciais, foram escolhidos dois mercados diferentes, de automóveis e de seguros. Primeiramente será realizada a modelagem matemática do problema através da taxa marginal de substituição com base no trabalho de Marques [3]. E, posteriormente, a aplicação desse modelo no mercado americano de automóveis, utilizando os dados apresentados no trabalho de Petrin [4] e no mercado brasileiro de seguros, cujos dados foram extraídos do site da Superintendência de Seguros Privados - SUSEP [5].

Com a compreensão do fenômeno de escolha neste ambiente de Microeconomia, cenários podem ser simulados com o intuito de se tentar fazer previsões a respeito das decisões de escolha, podendo inferir sobre o comportamento dos consumidores, e assim, trazer informações úteis para tomada de decisão de empresas atuantes no mercado.

$\mathrm{Na}$ Seção 2, tem-se a modelagem matemática via equações diferenciais, e na Seção 3, apresentam-se os dados utilizados para a aplicação no mercado Americano de automóveis, minivans e station wagons, além dos dados referentes às duas Seguradoras que atuam no mercado Brasileiro de seguros.

$\mathrm{Na}$ Seção 4 descreve-se a forma com que os dados foram incorporados na modelagem e, também, apresenta-se as curvas de indiferença obtidas para cada mercado em estudo. $\mathrm{Na}$ seção 5, interpreta-se os resultados obtidos analisando as seguradoras por meio das demonstrações financeiras. As conclusões deste trabalho encontram-se na Seção 6.

\section{Modelagem matemática}

Nesta seção, apresenta-se a equação diferencial ordinária utilizada para descrever o comportamento de um determinado produto, a medida que um outro produto concorrente passa a variar.

Dentro do ambiente da Microeconomia e em conjunto com o conceito de taxa de variação, a forma com que a variação de um produto afeta o produto concorrente é representada por uma derivação. 
A princípio considerando dois produtos $x$ e $y$ com uma mesma relação de preferência, ou seja, uma função de utilidade $y=y(x)$, e que a substituição entre os produtos ocorre suavemente, as preferências do consumidor, pela taxa marginal de substituição $\boldsymbol{\tau}$ de y para $\mathrm{x}$ [3], pode ser modelada por:

$$
\mathrm{dy} / \mathrm{dx}=-\boldsymbol{\tau}(\mathrm{x}, \mathrm{y}), \mathrm{x}>0 \text { e } \mathrm{y}>0,
$$

nota-se que, na equação (1), a variação do produto $\mathrm{x}$ afeta negativamente quando $\boldsymbol{\tau}$ assume valores positivos. Neste contexto, tem-se a interpretação de que consumidor está migrando do produto $\mathrm{y}$ para o produto $\mathrm{x}$, isto é, a preferência por $\mathrm{y}$ decai à medida que $\mathrm{x}$ aumenta.

Ao assumir que os dois produtos apresentam a mesma utilidade, pode-se inferir que $\boldsymbol{\tau}$ pertence a uma curva de indiferença, além de que considera-se não ser possível um indivíduo consumir ambos os produtos [3], ou seja, ao adquirir um produto fica impossibilitado de obter o outro. Assim sendo, uma classe de curvas de indiferença pode ser descrita por:

$$
\boldsymbol{\tau}(\mathrm{x}, \mathrm{y})=\mathrm{g}(\mathrm{x}) / \mathrm{x},
$$

em que $\mathrm{g}(\mathrm{x})$ é uma reta decrescente obtida através da análise dos dados do problema, por exemplo $\mathrm{g}(\mathrm{x})=-\mathrm{ax}+\mathrm{b}$, com $\mathrm{a}>0$ [6]. Dessa forma, com as duas equações temos uma equação diferencial ordinária que modela a preferência do consumidor por meio da taxa marginal de substituição e da curva de indiferença. Assumindo uma relação inicial entre esses produtos, temos o seguinte problema de valor inicial a ser investigado:

$$
\left\{\frac{d y}{d x}=\frac{-(-a x+b)}{x}=a-\frac{b}{x}, \quad y\left(x_{0}\right)=y_{0}\right\},
$$

em que $\mathrm{x}>0$ e $\mathrm{y}>0$.

Resolvendo o PVI (3) por separação de variáveis [7], a solução analítica é dado por:

onde $\mathrm{C}=\mathrm{y}_{0}-\mathrm{ax}_{0}+\mathrm{b} \cdot \ln (\mathrm{x})$.

$$
\mathrm{y}(\mathrm{x})=\mathrm{ax}-\mathrm{b} \cdot \ln (\mathrm{x})+\mathrm{C},
$$

Ao considerar um mercado específico, os valores de $\mathrm{a}, \mathrm{b}, \mathrm{y}_{0}$ e $\mathrm{x}_{0}$ são obtidos através dos dados dos produtos considerados e, em especial, as constantes a e b são obtida por um ajuste de curva [8], sendo a implementação computacional deste trabalho feita em Octave [9]. Para modelagem do problema, considerou-se um mercado Americano de automóveis e um mercado Brasileiro de seguros, e tais dados estão apresentados na próxima seção.

\section{Dados do mercado}

Para a aplicação de equações diferenciais ordinárias em Microeconomia, por meio da modelagem através de taxa marginal de substituição das preferências do consumidor, foram coletados dados disponíveis na literatura.

Visando analisar as preferências do consumidor no mercado Americano de automóveis, a base de dados utilizada está presente no trabalho de Petrin [4] que quantifica a entrada da minivan nos Estados Unidos.

Na Figura 1 foram plotados os dados desse mercado e ao observar tais dados percebe-se que as vendas do veículo de modelo station wagons sofre uma queda crescente em seu volume de vendas entre 1984 e 1992, após a inserção em 1984 do modelo minivan no 
mercado, sendo o sucesso da minivan estreitamente relacionado com o declínio do modelo mais antigo, conforme o gráfico apresentado na Figura 1.

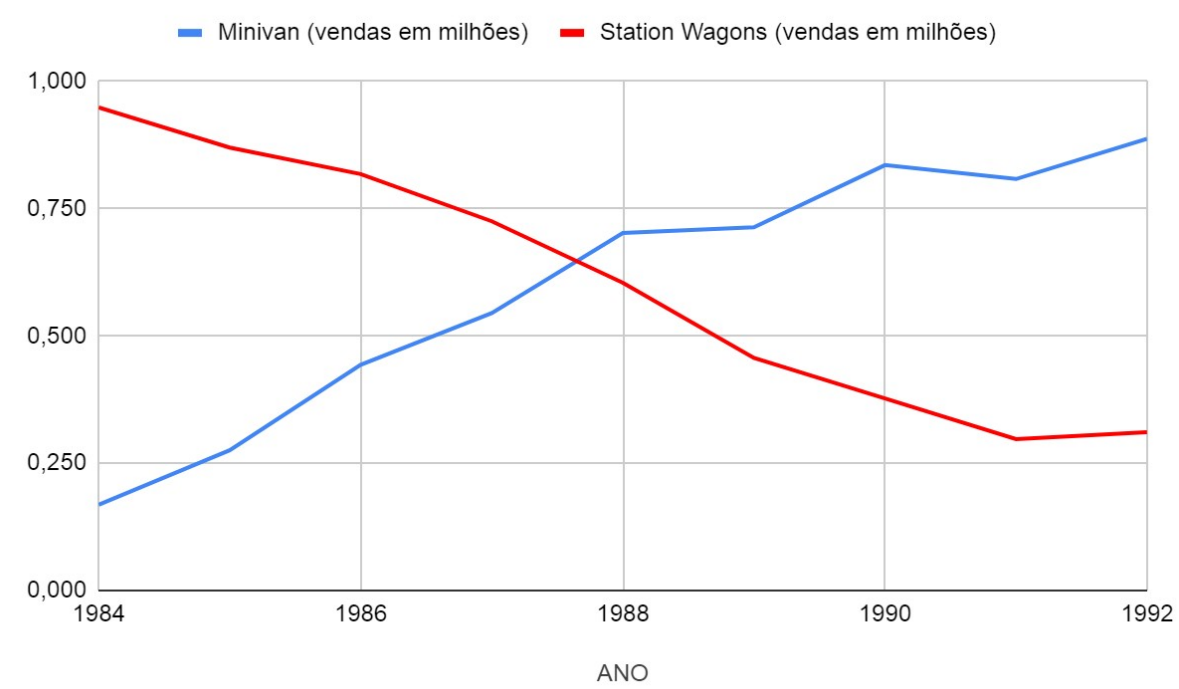

Figura 1 - Comparativo do volume de vendas entre 1984 e 1992

Nesse cenário, nota-se a preferência dos consumidores de automóveis pela minivan, ao longo dos anos, acompanhada pela queda do interesse de compra do modelo mais antigo. Considerando esses dois produtos, que fornecem a mesma utilidade aos indivíduos, é possível modelar esse problema, com base no modelo descrito anteriormente.

Analogamente, para aplicação do modelo matemático de preferências do consumidor, foram utilizadas informações do mercado securitário, com o objetivo de realizar inferências sobre o consumo dos produtos por ele ofertados. Para isso, foram coletados dados disponibilizados pelo seu órgão regulador, a Superintendência de Seguros Privados (SUSEP), que é responsável pelo controle e fiscalização dos mercados de seguro, previdência complementar aberta, capitalização e resseguro [5].

Através de seu Sistema de Estatísticas (SES), foram investigadas as entidades que oferecem seguro de Garantia de Obrigações Públicas que é definido, no Art. ${ }^{\circ}$ pela Circular $n^{\circ}$ 477 (SUSEP), como o seguro que tem por objetivo garantir o fiel cumprimento das obrigações assumidas pelo tomador perante o segurado.

$\mathrm{Na}$ análise desse ramo de seguro, foram verificados os valores relativos aos prêmios, definidos como a "importância paga pelo Segurado ou estipulante/proponente à Seguradora para que esta assuma o risco a que o Segurado está exposto" (Circular SUSEP 306/05), com o objetivo de identificar a atuação das seguradoras nesse mercado. Foi constatada a predominância de duas seguradoras, a Swiss Re Corporate Solutions Brasil Seguros S.A. e a Junto Seguros S.A., as duas principais competidoras no mercado de seguros de Garantia de Obrigações Públicas, entre 2015 e 2018.

É importante ressaltar que embora essas seguradoras sejam as principais desse ramo, elas também atuam em outros setores do mercado securitário, sendo a Garantia de Obrigações Públicas um ramo específico. A Junto S.A. atua em fiança locatícia e quatro outros segmentos de seguro garantia, enquanto que a Swiss S.A. opera no ramo de seguro rural, patrimonial, riscos financeiros, transportes, DPVAT, responsabilidades, aeronáuticos, marítimos e riscos especiais. 
Dentro do período analisado, foi possível observar uma redução nos prêmios emitidos pela Junto S.A., em detrimento ao aumento na emissão de prêmios pela Swiss S.A. Esse movimento do mercado pode ser representado pela Figura 2.

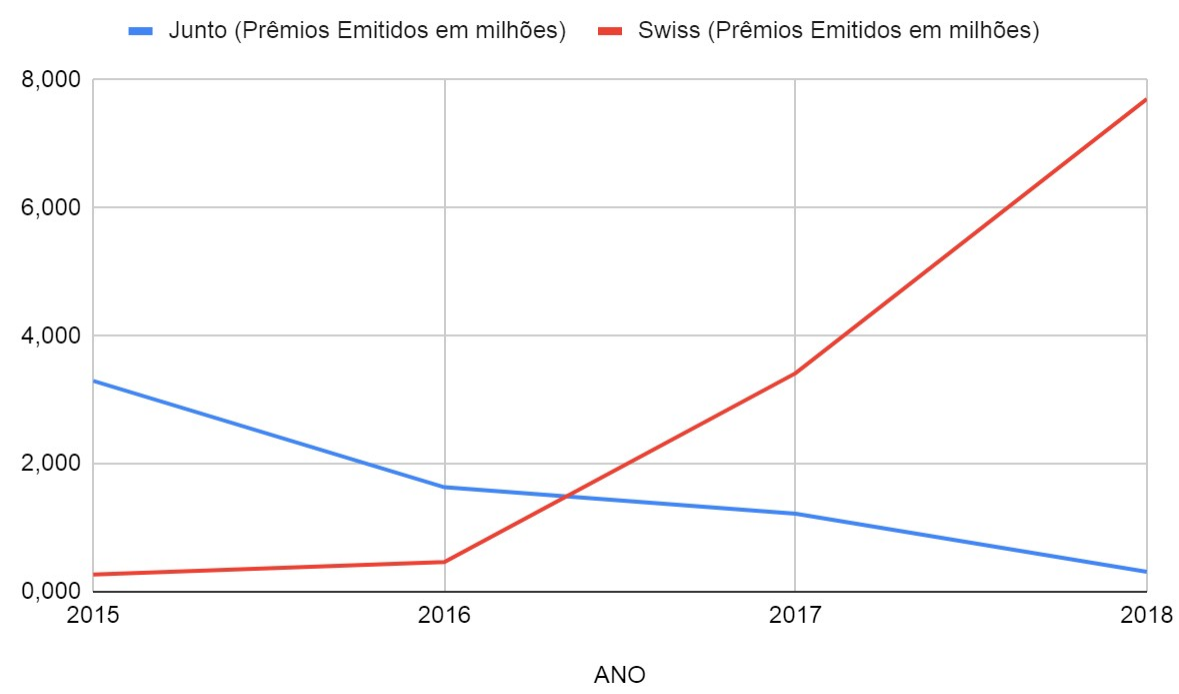

Figura 2 - Comparativo do volume de prêmios entre 2015 e 2018

Tendo em vista que ambas as seguradoras oferecem ao mercado produtos securitários de mesma utilidade, visto que os dois protegem seus segurados do risco oriundo de obrigações públicas adquiridas, nota-se uma preferência dos consumidores desse mercado pelo produto fornecido pela Swiss S.A., em detrimento da Junto S.A. Sendo possível investigar esse problema através do modelo matemático proposto.

\section{Resultados}

Nessa seção, serão apresentados os resultados obtidos através da aplicação da modelagem matemática nos problemas propostos, tanto no mercado automotivo, como no mercado securitário. Através dos dados da seção 3, foi possível obter a constante $\mathrm{C}$ e a condição inicial necessárias para a definição da equação diferencial ordinária, conforme apresentado a seguir.

\subsection{Mercado de automóveis}

Para a obtenção da constante $\mathrm{C}$, foi realizada a regressão linear do volume de vendas dos dois produtos minivans e station wagons, em milhões de unidades, entre os anos de $1984 \mathrm{e}$ 1992, ver Figura 3. 
Figura 3 - Regressão Linear do Volume de Vendas

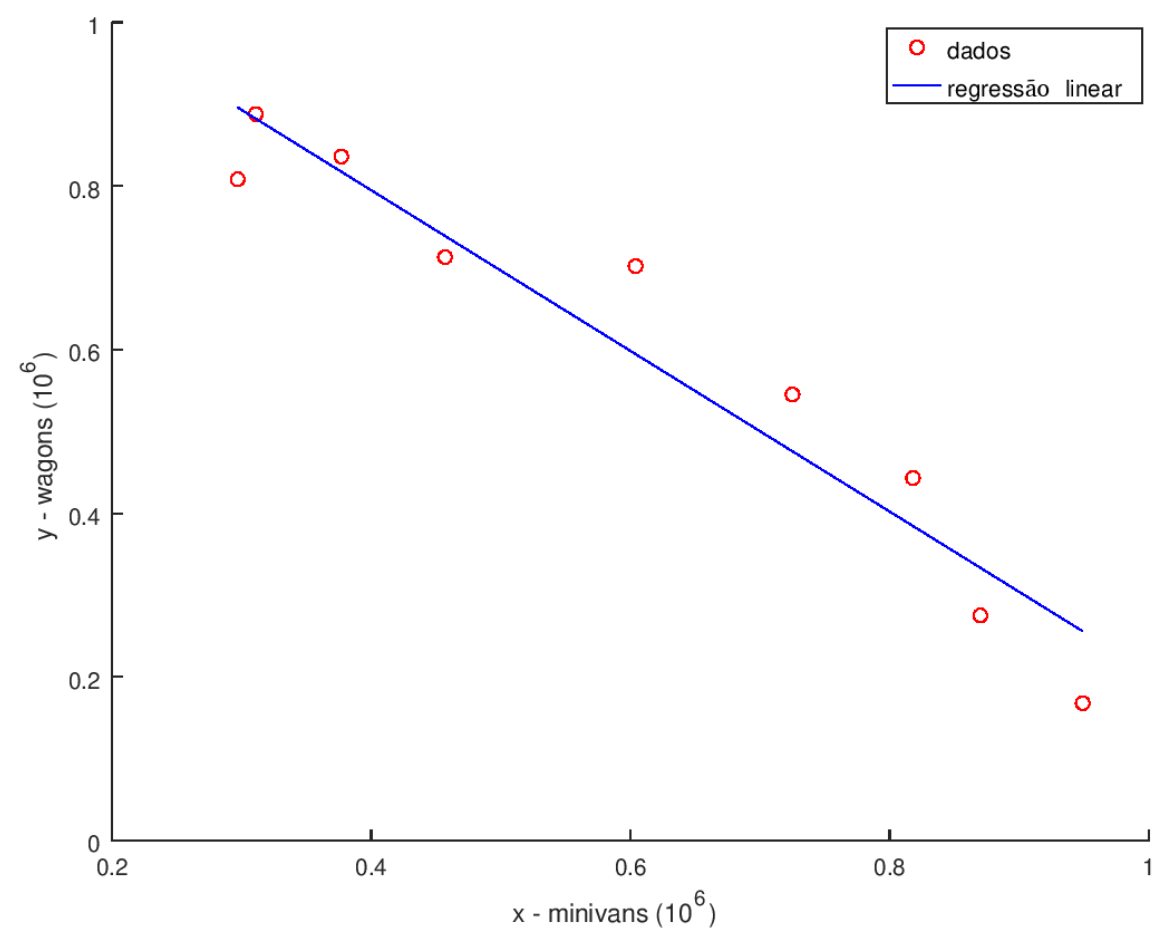

O polinômio obtido foi $(\mathrm{y}=-0,98186 . \mathrm{x}+1,1875)$. Portanto, o valor das constantes a e $\mathrm{b}$ é dada por:

$$
\mathrm{a}=0,98186 \text { e } \mathrm{b}=1,1875 \text {. }
$$

Dessa maneira, é possível descrever a equação diferencial ordinária que modela o problema da seguinte forma.

$$
\frac{d y}{d x}=0,98186-\frac{1,1875}{x},
$$

A condição inicial estabelecida baseou-se nos dados históricos de vendas. Foram consideradas a média amostral das vendas totais dos dois produtos (em milhões), durante o período adotado para análise, isto é, $x_{0}=\bar{x}$ e $y_{0}=\bar{y}$. Sendo assim,

$$
\mathrm{y}(0,60089)=0,59756
$$

A partir da equação diferencial ordinária obtida, foi possível realizar a modelagem do problema proposto, resolvendo a equação analiticamente, junto com os valores de a e b, e a condição inicial, sendo a curva de indiferença resultante para o problema de valor inicial:

$$
y=0,9819 x-1,1875 \cdot \ln (x)-0,5973
$$

cuja função está graficada na Figura 4. Com essa equação foi possível estudar o comportamento de preferência do mercado de minivans, entre duas marcas x e y. 


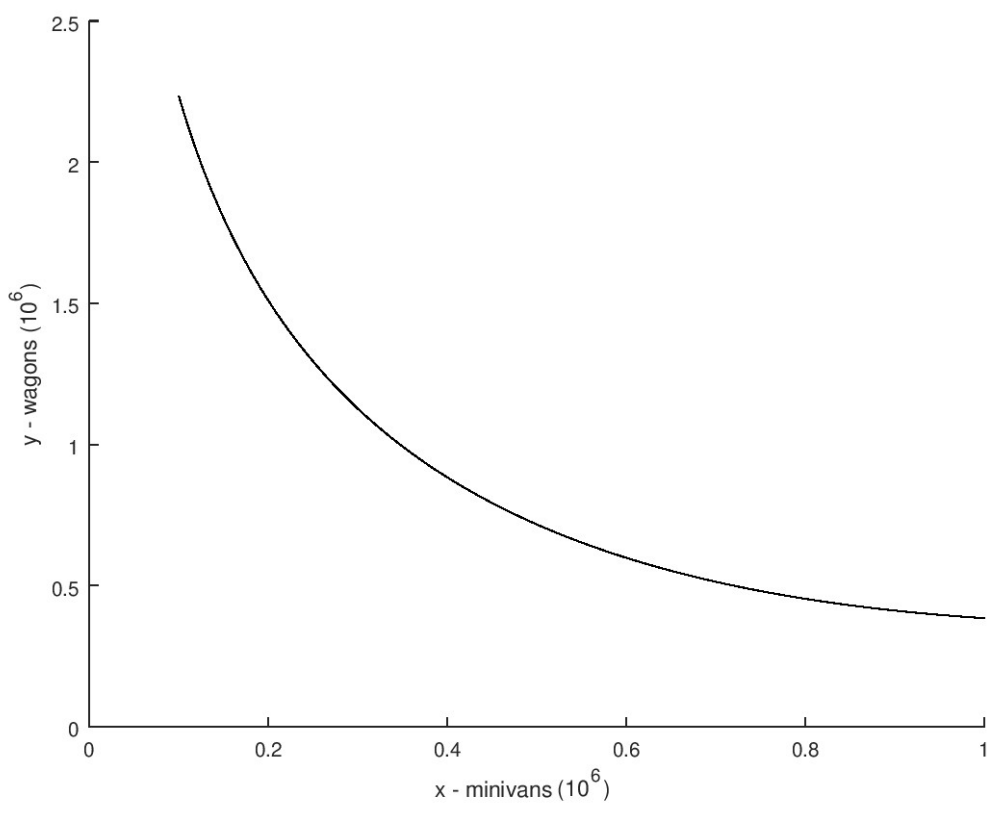

Figura 4 - Curva de indiferença de Minivan e Station wagons

Na Figura 4 percebe-se que a partir de um determinado momento, $x$ precisa vender uma grande quantidade para conseguir impactar nas vendas de $\mathrm{y}$, entretanto, nos anos logo após a condição inicial, a vendas de $\mathrm{y}$ rapidamente diminuem à medida que as vendas de $\mathrm{x}$ aumentam. Dessa forma, pode-se concluir que, de início, as vendas de station wagons tiveram um grande impacto nas vendas de minivans, porém, ao passar do tempo, as vendas de station wagons não tem mais um grande impacto nas vendas de minivans.

A preferência dos consumidores pela minivan pode ter sido causada por certas características presentes nesse modelo, tais como: um maior espaço interno, através da altura, facilitando a movimentação de dentro do veículo, além de portas traseiras deslizantes, diferenciais desse novo modelo lançado em 1984, em comparação com a station wagons [4]. Desse modo, nota-se um interesse maior do mercado por espaços internos mais generosos, além de maior praticidade no embarque e desembarque de passageiros.

Logo, a empresa que oferece o produto station wagon precisava alterar suas práticas de vendas para que seu produto voltasse a ter um grande impacto nas vendas de minivans, caso contrário, seriam necessárias muitas vendas de station wagons para restringir a preferência do consumidor por minivans. O comportamento pode ser extrapolado para além do ano de 1992, para isso, basta considerar o domínio de solução além dos valores de $\mathrm{x}$ apresentados na Figura 4.

\subsection{Mercado de seguros}

Visando analisar as preferências do consumidor no mercado de seguros descrito na seção 3, considerou-se os dados da Figura 2. Nesta análise, seguiu-se os mesmos procedimentos da Seção 4.1, logo a regressão linear obtida do volume de prêmios emitidos da seguradora Junto, em detrimento aos prêmios emitidos pela seguradora Swiss foi $\mathrm{y}=-0,3024 \mathrm{x}+2,5104$, ver Figura 5. 


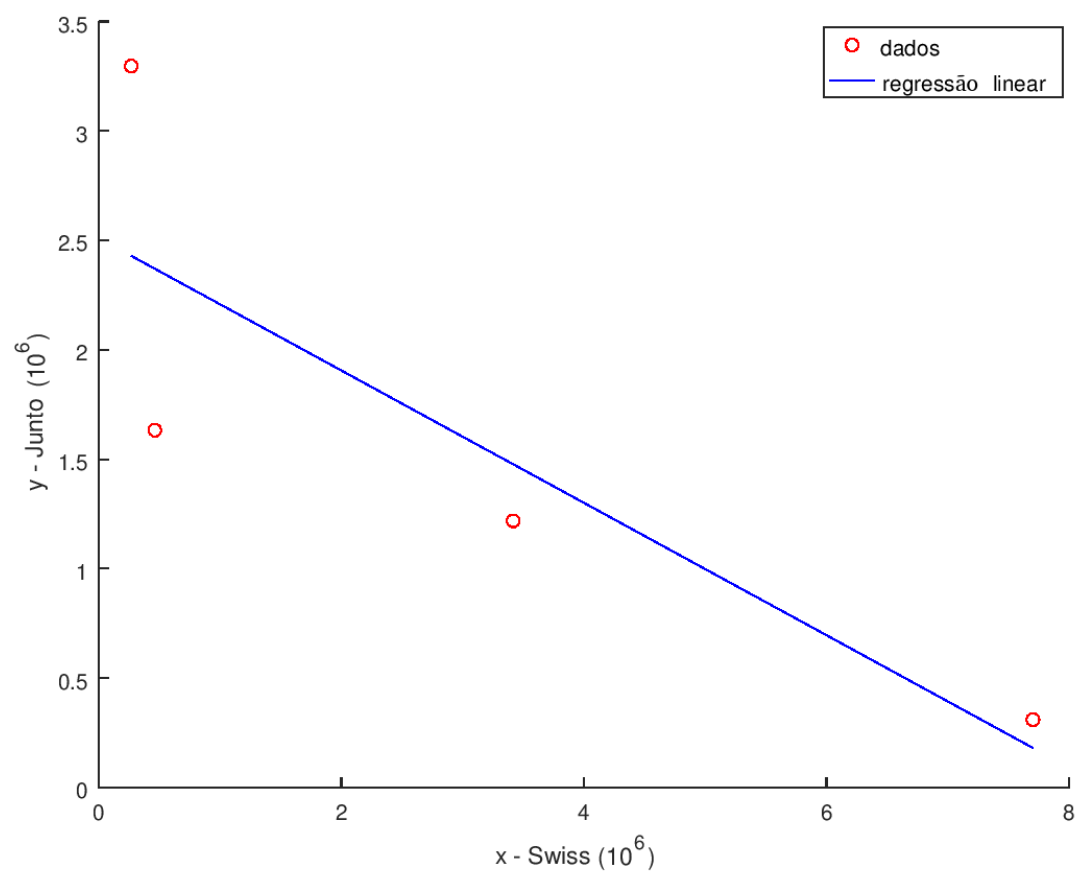

Figura 5 - Regressão linear dos dados das seguradoras. No eixo x encontra-se a quantidade de prêmios emitidos pela Swiss S.A. e no eixo y a quantidade emitida pela Junto.

Com as informações obtidas, foi possível construir a equação diferencial ordinária que modela o problema:

$$
\frac{d y}{d x}=0,3024-\frac{2,5104}{x}
$$

Para determinar a condição inicial, foram consideradas a média de prêmios emitidos pela seguradora Junto S.A. como $\bar{x}$ e $\bar{y}$ a média amostral relativa à seguradora Swiss S.A. Sendo assim,

$$
y(2,9621)=1,6147
$$

Resolvendo analiticamente o PVI definido pelas equações (9) e (10), a solução obitida foi:

$$
y=0,3024 x-2,5104 \ln (x)+3,4451 \text {, }
$$

em que o gráfico dessa curva de indiferença está representada na Figura 6.

A Figura 6 apresenta um comportamento similar ao estudado na seção 4.1, isto é, de início, a seguradora Swiss S.A. tem um forte impacto na Junto S.A., entretanto esse comportamento é apenas inicial. Ao passar do tempo, a empresa Swiss S.A. atinge cada vez menos sua concorrente e, se quiser modificar esse cenário, precisará intensificar suas estratégias de vendas para maior efeito sobre a opção do consumidor pela Junto S.A. 


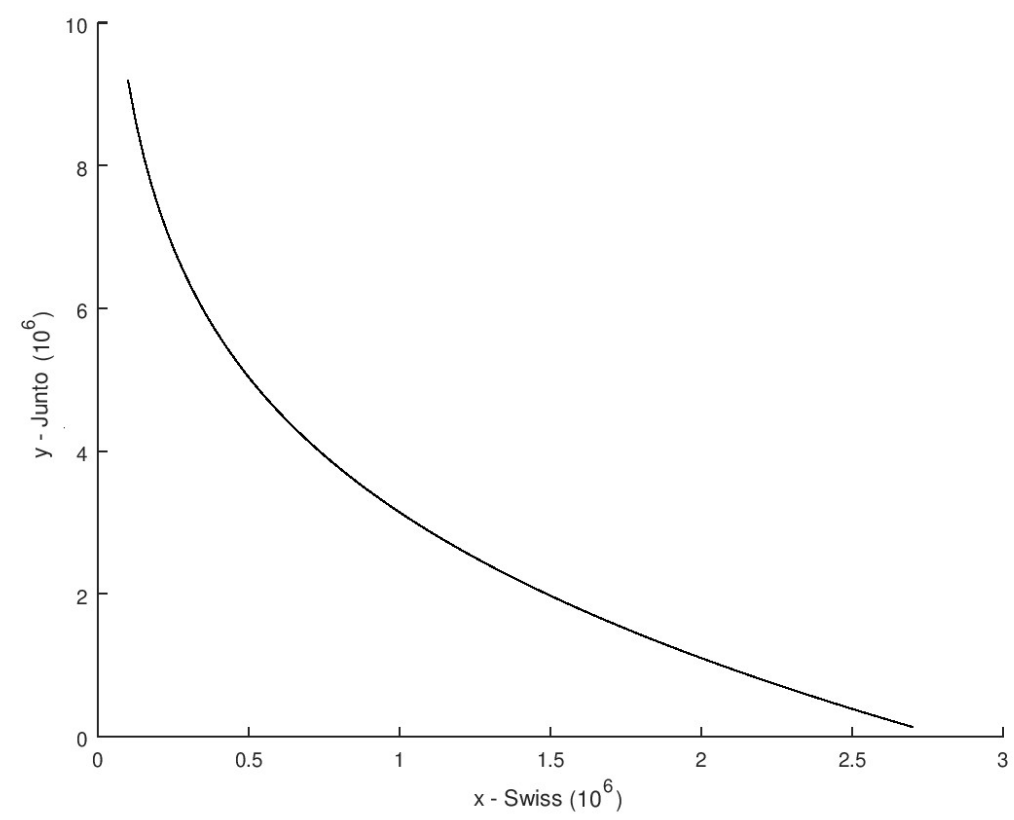

Figura 6 - Curva de indiferença obtida através do PVI aplicado aos dados das seguradoras. No eixo x encontra-se a quantidade de prêmios emitidos pela Swiss S.A. e no eixo y a quantidade emitida pela Junto.

A preferência dos consumidores pela Swiss S.A. pode estar relacionada a três fatores principais atrativos no mercado, sendo eles: menores valores de prêmios, uma maior qualidade no atendimento e o pagamento ágil na ocorrência de um sinistro. Também há a possibilidade de migração da Junto S.A. a outros ramos securitários, fazendo com que reduza aos poucos a emissão de apólices de seguros nesse setor.

Nesse cenário, através de inferências sobre os prêmios emitidos futuros das seguradoras desse ramo, admitindo a continuidade das preferências do consumidor modelado neste trabalho, há um alerta às empresas atuantes nesse ramo para decisões estratégicas visando a expansão no mercado.

\section{Análise das seguradoras}

A fim de verificar com maior profundidade o impacto da seguradora Swiss S.A. no desempenho da Junto S.A., em especial, no que se refere ao ramo de Garantia de Obrigações Públicas, foram observadas as demonstrações financeiras publicadas [5], de ambas as seguradoras, em 2017 e 2018.

A seguradora Junto, embora tenha emitido cada vez menos prêmios no ramo de Garantia de Obrigações Públicas, tem apresentado bons resultados, em continuidade aos dois exercícios anteriores, com um lucro líquido consistente entre 2017 e 2018. Enquanto vem lidando com valores decrescentes de prêmios emitidos líquidos, referente a soma de todos os ramos nos quais atua, desde 2016.

O lucro auferido nesse período foi influenciado pela redução nas despesas operacionais provenientes das atividades da entidade, relacionadas à sinistralidade e ao custo de aquisição diferido. Essas despesas se referem a gastos oriundos da concretização do risco e da emissão da apólice de seguro, além de outros dispêndios, tais como: despesas administrativas, tributárias e outras despesas operacionais. 
Em 2017, tais despesas subtraíam $66 \%$ do total de prêmios ganhos, reduzindo para $59 \%$ em 2018. Em contrapartida, houve um aumento de outros tipos de despesas, de resseguro e financeiras, que tiveram seus efeitos anulados, mantendo seu lucro com uma margem líquida de $13 \%$.

Dentro desse período, observou-se um aumento da emissão de prêmios nos ramos de fiança locatícia e garantia de obrigações privadas em que a Junto S.A. atua, acompanhado pela diminuição nos outros ramos que a seguradora opera, em especial no ramo de Garantia de Obrigações Públicas que foi o ramo que sofreu a maior redução quanto à emissão de prêmios, em comparação com todos os outros segmentos.

É possível inferir, portanto, que a seguradora Junto S.A. embora tenha sido impactada pela Swiss S.A., tendo perdido competitividade e mercado no ramo de Garantia de Obrigações Públicas para a sua concorrente, e mesmo que lide com uma redução de prêmios emitidos tanto nesse ramo específico, como em outros ramos (prêmios emitidos líquidos), ela tem conseguido manter seus lucros, ao reduzir gastos na área operacional e ao investir em outros ramos nos quais já é atuante.

Outro fator importante, verificado através das demonstrações, quanto ao descompasso entre as seguradoras na emissão de prêmios, pode estar relacionado ao fato das empresas estarem classificando os valores de prêmios em ramos securitários indevidos, uma vez que há outros ramos em que elas operam que têm pequena diferenciação de nomenclatura de Garantia de Obrigações Públicas.

\section{Conclusões}

Neste trabalho, realizou-se a modelagem matemática de preferência de dois mercados, a saber, de automóveis e de seguros, através de equações diferenciais ordinárias. Com o PVI (3) definido para cada segmento através dos dados de mercado, foram possíveis de se obter soluções analíticas que fornecem a curva de indiferença para cada um deles. Com tais soluções, foram realizadas análises do comportamento da variação de um produto, com relação ao produto concorrente.

Ao analisar as soluções obtidas nas seções 4.1 e 4.2 foi possível analisar as preferências do consumidor, observando o comportamento do mercado. Com isso, possibilita-se auxiliar às entidades a preverem seu direcionamento, fundamentando seus processos decisórios.

No mercado de automóveis, com relação à curva de indiferença obtida, que representa as preferências entre os dois produtos, verifica-se uma taxa marginal de substituição decrescente, ou seja, cada vez os consumidores desse mercado estavam menos dispostos a abrir mão de uma unidade de station wagons por unidades de minivan. Desse modo, as entidades comercializadoras de minivans apresentaram resultados relativos a vendas de minivans cada vez menores, precisando investir na comercialização de outros produtos, visando a preservação de seus lucros.

A taxa de queda das vendas da station wagons estar menor, não significa que os consumidores estivessem voltando a consumir esse produto, pelo contrário, indica que aos poucos sua concorrente dominaria o mercado (Figura 4). Entretanto, a medida que o tempo passava, seriam necessários muito mais vendas de minivans para impactar a venda de station wagons. Caso a empresa de station wagons quisesse voltar a ter um grande impacto nas vendas de minivans, suas estratégias de vendas precisariam ser revistas.

No mercado de seguros, obteve-se um comportamento análogo ao mercado de automóveis, de início a seguradora Swiss S.A. ocasionou um grande impacto nas vendas de seguros da empresa Junto S.A., entretanto, à medida que o tempo evoluiu, ele foi ficando cada vez menor, conforme a Figura 6. Ademais, ao realizar uma análise das demonstrações 
financeiras da Junto S.A., foi possível aprofundar o entendimento sobre as motivações e impactos das mudanças nas emissões de prêmios entre as duas seguradoras operantes no ramo de Garantia de Obrigações Públicas.

Portanto, com a modelagem matemática descrita nesse trabalho, foi possível extrair a curva de indiferença entre dois produtos de dois mercados diferentes e, dessa forma, fazer inferências sobre o comportamento do mercado consumidor no mercado de automóveis e no mercado de seguros, além de permitir um maior entendimento preditivo acerca de mercados distintos, permeando melhores condições para a tomada de decisão dos usuários, sendo eles internos ou externos às empresas.

\section{Agradecimentos}

A primeira autora agradece à Universidade Federal de São Paulo pela bolsa institucional concedida. E também, juntamente como segundo autor, agradecem ao prof. Dr. Francisco Marcelo Monteiro da Rocha pelas indicações de referências relativas ao conteúdo desenvolvido neste trabalho.

\section{Referências}

[1] PINDYCK, R. S.; RUBINFELD, D. L. Microeconomia. 7. ed. São Paulo: Pearson, 2010.

[2] SILVA, G. B; GARCIA, R. O; COSTA, J. A. Análise comportamental de preferências via equações diferenciais ordinárias. In: ENCONTRO REGIONAL DE MATEMÁTICA APLICADA E COMPUTACIONAL, 6., 2019, Bauru. Caderno de trabalhos completos e resumos [...]. Bauru: Unesp, Faculdade de Ciências, 2019. p. 428-429. Disponível em: https://www.fc.unesp.br/\#!/departamentos/matematica/eventos2341/ermac-2019/caderno-detrabalhos-e-resumos/. Acesso em: 12 ago. 2019.

[3] MARQUES, J. An application of ordinary differential equation in Economics: modeling consumer's preferences using marginal rates of substitution. In: INTERNACIONAL CONFERENCE ON MATHEMATICAL METHODS, COMPUTATIONAL TECHNIQUES AND INTELLIGENT SYSTEMS, 16., 2014, Lisboa. Anais [...]. Lisboa: [s.n.], 2014, p. 4653.

[4] PETRIN, A. Quantifying the benefits of new products: the case of the Minivan. Journal of Political Economy, v. 110, n. 4, p. 705-729, 2002.

[5] SUPERINTENDÊNCIA DE SEGUROS PRIVADOS (SUSEP). 2019. Disponível em: http://www.susep.gov.br/. Acesso em: 25 abr. 2019.

[6] BELLEFLAMME, P.; PEITZ, M. Industrial organization: markets and strategies. Cambridge: Cambridge University Press, 2010.

[7] BOYCE, W. E.; DIPRIMA, R. C. Equações diferenciais elementares e problemas de valores de contorno. 10. ed. Rio de Janeiro: LTC, 2017.

[8] RUGgiero, M. A. G.; LOPES, V. L. R. Cálculo numérico: aspectos teóricos e computacionais. 2. ed. São Paulo: Pearson, 1996. 
[9] GNU OCTAVE. 2019. Disponível em: https://www.gnu.org/software/octave/. Acesso em: 8 maio 2019. 